\title{
Mechanism of Poly(acrylic acid) Acceleration of Antithrombin Inhibition of Thrombin: Implications for the Design of Novel Heparin Mimics
}

\author{
Bernhard H. Monien, ${ }^{\dagger}$ Kai I. Cheang,, and Umesh R. Desai ${ }^{*}, \dagger$, \\ Departments of Medicinal Chemistry and Pharmacy and Institute for Structural Biology and Drug Discovery, \\ Virginia Commonwealth University, Richmond, Virginia 23298
}

Received April 19, 2005

\begin{abstract}
The bridging mechanism of antithrombin inhibition of thrombin is a dominant mechanism contributing a massive $\sim 2500$-fold acceleration in the reaction rate and is also a key reason for the clinical usage of heparin. Our recent study of the antithrombin-activating properties of a carboxylic acid-based polymer, poly(acrylic acid) (PAA), demonstrated a surprisingly high acceleration in thrombin inhibition (Monien, B. H.; Desai, U. R. J. Med. Chem. 2005, 48, 1269). To better understand this interesting phenomenon, we have studied the mechanism of PAAdependent acceleration in antithrombin inhibition of thrombin. Competitive binding studies with low-affinity heparin and a heparin tetrasaccharide suggest that PAA binds antithrombin in both the pentasaccharide- and the extended heparin-binding sites, and these results are corroborated by molecular modeling. The salt-dependence of the $K_{\mathrm{D}}$ of the PAA-antithrombin interaction shows the formation of five ionic interactions. In contrast, the contribution of nonionic forces is miniscule, resulting in an interaction that is significantly weaker than that observed for heparins. A bell-shaped profile of the observed rate constant for antithrombin inhibition of thrombin as a function of PAA concentration was observed, suggesting that inhibition proceeds through the "bridging" mechanism. The knowledge gained in this mechanistic study highlights important rules for the rational design of orally available heparin mimics.
\end{abstract}

\section{Introduction}

The process of fibrin formation involves the sequential activation of trypsin-like serine proteinases that possess interesting similarities among them, yet simultaneously display both subtle and ingenuous differences. Fibrin formation is primarily regulated by antithrombin, a plasma glycoprotein and member of the serpin (serine proteinase inhibitor) super family of proteins. ${ }^{1,2}$ Antithrombin regulates fibrin formation by primarily inhibiting three procoagulant proteinases, factor IXa, factor $\mathrm{Xa}$, and thrombin. However, antithrombin is a rather poor inhibitor of these procoagulant enzymes. Whereas most serpins inhibit their target proteinases at rates reaching the diffusion limit $\left(10^{7}-10^{8} \mathrm{M}^{-1} \mathrm{~s}^{-1}\right)$, antithrombin inhibition of factor Xa and thrombin is nearly 1000-10 000-fold slower.

The lethargic reaction is greatly accelerated in the presence of heparin, a natural sulfated linear polysaccharide, which affords a surface for the rapid assembly of ternary antithrombin-proteinase complexes. ${ }^{2-7}$ Under in vivo conditions, this "bridging mechanism" is responsible for most of the accelerating effect of heparin on the inhibition of all three proteinases. In another mechanism, the binding of a specific five-residue sequence in heparin, pentasaccharide DEFGH (Figure 1), induces a conformational change in the serpin that

\footnotetext{
* Address for correspondence: Department of Medicinal Chemistry, Virginia Commonwealth University, 410 N. 12th Street, P.O. Box 980540, Richmond, VA 23298-0540. Ph: 804-828-7328; fax: 804-8273664; e-mail: urdesai@vcu.edu.

${ }^{\dagger}$ Department of Medicinal Chemistry.

\$ Department of Pharmacy.

$\S$ Institute for Structural Biology and Drug Discovery.
}

A)

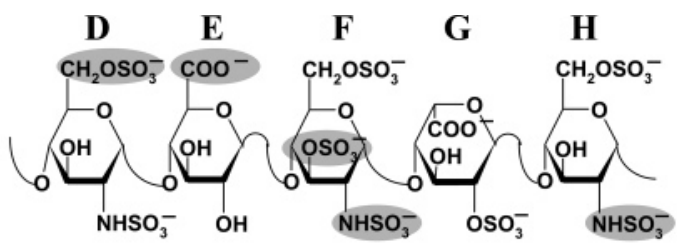

B)

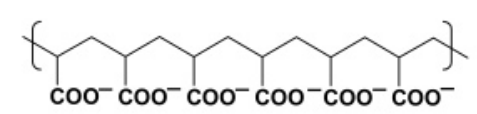

\begin{tabular}{lccccc} 
& $\boldsymbol{M}_{\boldsymbol{R}}$ & Polydispersity & Monomers & $\boldsymbol{K}_{\boldsymbol{D}}$ & Acceleration \\
\hline & $(k D a)$ & & $(\#)$ & $(\mu M)$ & $\left(k_{A C T^{\prime}} / k_{U N C A D}\right)$ \\
PAA830 & 0.8 & 1.6 & $\sim 12$ & nd & 118 -fold \\
$\boldsymbol{P A A 1 5 3 0}$ & 1.5 & 1.1 & $\sim 21$ & 180 & 114 -fold \\
$\boldsymbol{P A A 2 2 8 0}$ & 2.3 & 1.7 & $\sim 32$ & 96 & 259 -fold \\
$\boldsymbol{P A A 3 4 5 0}$ & 3.5 & 1.6 & $\sim 48$ & 91 & 579 -fold \\
$\boldsymbol{P A A 6 2 0 0}$ & 6.2 & 1.3 & $\sim 87$ & 34 & 1109 -fold \\
\hline
\end{tabular}

Figure 1. Structures of high affinity pentasaccharide DEFGH sequence of heparin (A) and PAA (B). Sulfate and carboxylate groups in A contributing to the high affinity for antithrombin are shown in gray ovals. The table corresponding to PAAs summarizes their important properties: $M_{\mathrm{R}}=$ average molecular weight; nd $=$ not determined; $K_{\mathrm{D}}$ refers to antithrombin binding affinity at $\mathrm{pH} 7.4, I 0.05$, and $25^{\circ} \mathrm{C}$; acceleration refers to the increase in antithrombin inhibition of thrombin due to PAA at $\mathrm{pH} 7.4$, I 0.05 , and $25{ }^{\circ} \mathrm{C}$. Reprinted from ref 13 with permission. Copyright 2005 American Chemical Society.

enhances its recognition of the proteinase, resulting in 50 an acceleration in the inhibition rate. ${ }^{6,8}$ This mechanism 51 is called the "conformational activation" mechanism, 52 which causes an $\sim 500$ - and 300-fold acceleration in the 53 inhibition of factor IXa and factor Xa, respectively. The 54 bridging mechanism with full-length heparin polymer, $\quad 55$ in turn, induces an additional $\sim 600$ - and 170-fold 56 
acceleration in the inhibition of these two enzymes, respectively. In contrast, thrombin is inhibited only 1.7 times faster through the conformational activation mechanism but is inhibited $\sim 2500$ times faster using the bridging mechanism. ${ }^{3,7}$

Unfractionated heparin (UFH), obtained from animal sources, has been used clinically as an anticoagulant since the 1930s, yet heparin therapy suffers because of an increased risk for bleeding, heparin-induced thrombocytopenia (HIT), and patient-to-patient response variability. ${ }^{9,10}$ The growing understanding of antithrombinheparin interactions at a molecular level has supported the development of newer agents with fewer side effects during the last two decades. ${ }^{11}$ Reduction in molecular weight and the polydispersity of the highly heterogeneous heparin preparation, as found in low molecular weight heparins (LMWHs), were found to diminish the adverse effects. Recently, a synthetic derivative of DEFGH, fondaparinux, was introduced clinically. ${ }^{12}$ Despite these advances, heparin anticoagulant therapy continues to suffer because of the risk for bleeding. In addition, the intricate and difficult synthesis of oligosaccharides makes fondaparinux treatment less cost-effective. Finally, the highly anionic character of these sulfated molecules, as well as the presence of heparanase in the gastrointestinal (GI) lining, makes oral activity nearly impossible.

Three strategies can be considered for developing orally bioavailable antithrombin activators, including (i) the design of heparin mimics completely devoid of sulfate groups, (ii) the development of sulfate-protecting groups that hydrolyze in blood, and (iii) the development of micellar delivery agents as absorption promoters. We reasoned that heparin mimics based only on carboxylic acid groups and devoid of sulfate groups might facilitate a relatively easy prodrug approach for passage through the GI lining.

Our recent study of the antithrombin-activating properties of a carboxylic acid-based polymer, poly(acrylic acid) (PAA, Figure 1), demonstrated a surprisingly high acceleration of thrombin and factor Xa inhibition. ${ }^{13}$ The PAA molecules, having no resemblance to a heparinlike polysaccharide scaffold but possessing a charge density comparable to heparin, increased the secondorder rate constant of factor $\mathrm{Xa}$ and thrombin inhibition several hundred-fold (Figure 1). This was in striking contrast to other carboxylate polymers, poly(Asp) and poly (Glu), that have been known to display poor antithrombin activation properties. ${ }^{14}$ To better understand this interesting phenomenon with the goal of designing a functional mimic of heparin that may facilitate oral delivery, we have studied the mechanism of PAAdependent acceleration in antithrombin inhibition of thrombin. The results suggest considerable similarity with the mechanism of heparin activation of antithrombin, yet they highlight a number of important differences. Overall, the results indicate that, despite the excellent antithrombin-activating property of PAAs, these carboxylate-based polymers are unlikely to be useful in vivo. Yet, the molecular interaction studies highlight principles for the design of orally bioavailable nonpolysaccharide heparin mimics.

\section{Materials and Methods}

Proteins and Chemicals. Human antithrombin was obtained from Molecular Innovations (Southfield, MI), and factor $\mathrm{Xa}$ and thrombin were obtained from Haematologic Technologies (Essex Junction, VT). The proteins were used as purchased, and stock solutions [prepared in $20 \mathrm{mM}$ sodium phosphate buffer, $\mathrm{pH} 7.4$, containing $100 \mathrm{mM} \mathrm{NaCl}$ (antithrombin and thrombin) or in $5 \mathrm{mM}$ MES buffer, $\mathrm{pH} 6.0$, containing $20 \mathrm{mM} \mathrm{NaCl}$ (factor Xa)] were stored at $-78{ }^{\circ} \mathrm{C}$. The low-affinity heparin (LAH, average molecular weight $\left(M_{\mathrm{R}}\right)$ $=7900 \pm 10 \% \mathrm{Da}$ ) and tetrasaccharide $\mathrm{EFGH}^{\prime \prime}$ used were a generous gift from Professor Steven T. Olson of the University of Illinois-Chicago. Chromogenic substrates Spectrozyme TH (thrombin) and Spectrozyme FXa (factor Xa) were obtained from American Diagnostica (Greenwich, CT). PAAs, with average molecular weights of 830, 1500, 2280, 3450, and 6200 Da, were obtained from either American Polymer Standards (Mentor, OH) or Polymer Source (Dorval, Canada). 2-( $p$ Toluidino)naphthalene-6-sulfonic acid (TNS) was obtained from Aldrich Chemicals (Milwaukee, WI). All other chemicals were reagent grade from either Aldrich Chemicals (Milwaukee, WI) or Fisher (Pittsburgh, PA) and were used without further purification.

Thrombin Inhibition with Antithrombin and PAA2280. PAA-promoted thrombin inhibition was studied by monitoring the pseudo-first-order rate constant $\left(k_{\mathrm{OBS}}\right)$ over a wide range of PAA concentrations $(100 \mathrm{nM}-2 \mathrm{mM})$ with $300 \mathrm{nM}$ antithrombin (AT) and $30 \mathrm{nM}$ thrombin (T).5,7,13 The reactions were carried out in PEG20000-coated cuvettes $(\mathrm{PEG}=$ polyethylene glycol) at $25{ }^{\circ} \mathrm{C}$ in $20 \mathrm{mM}$ sodium phosphate buffer, $\mathrm{pH} 6.0$, containing $20 \mathrm{mM} \mathrm{NaCl}, 0.1 \mathrm{mM}$ EDTA, and 0.1\% PEG8000. The $k_{\mathrm{OBS}}$ at each $[\mathrm{PAA}]_{0}$ was calculated from the exponential decay of the residual enzyme activity as a function of time. The second-order rate constant $\left(k_{\mathrm{CAT}}\right)$ for the inhibition of thrombin was determined by fitting the dependence of $k_{\mathrm{OBS}}$ on $[\mathrm{PAA}]_{0}$ with eq 1 :

$k_{\mathrm{OBS}}=k_{\mathrm{UNCAT}}[\mathrm{AT}]_{0}+k_{\mathrm{ACT}}[\mathrm{AT} / \mathrm{PAA}] \frac{K_{\mathrm{T}, \mathrm{PAA}}}{K_{\mathrm{T}, \mathrm{PAA}}+[\mathrm{PAA}]_{0}}$

in which [AT/PAA] was obtained using the quadratic equilibrium binding, $K_{\mathrm{T}, \mathrm{PAA}}$ and $K_{\mathrm{AT} \text {,PAA }}$ are dissociation constants for thrombin-PAA and antihrombin-PAA interaction, respectively, and $k_{\mathrm{UNCAT}}$ and $k_{\mathrm{ACT}}$ are the second-order rate constants for the reaction of antithrombin with thrombin and for the reaction of the antithrombin-PAA complex with thrombin, respectively. The second-order rate constant $k_{\mathrm{UNCAT}}$ and the equilibrium dissociation constant for antithrombin-PAA interaction $\left(K_{\mathrm{AT}, \mathrm{PAA}}\right)$ in eq 1 were determined independently. ${ }^{13}$

Equilibrium Binding Studies of Antithrombin-PAA Interactions. The equilibrium dissociation constant $\left(K_{\mathrm{D}}\right)$ of the interaction between PAA and antithrombin was determined through fluorescence titrations using a nonspecific probe, TNS $\left(\lambda_{\mathrm{EX}}=330 \mathrm{~nm}, \lambda_{\mathrm{EM}}=432 \mathrm{~nm}\right)$. Titrations of $0.95-$ $1.9 \mu \mathrm{M}$ antithrombin and $5.5 \mu \mathrm{M}$ TNS were carried out with 1-20 mM PAA in $20 \mathrm{mM}$ sodium phosphate buffer, $\mathrm{pH} 6.0$, containing $0.1 \mathrm{mM}$ EDTA and $0.1 \%$ PEG8000, at $25^{\circ} \mathrm{C}$ and in the presence of various concentrations of $\mathrm{NaCl}^{15,16}$ The fluorescence intensity of the sample at $432 \mathrm{~nm}$ decreases steadily as PAA concentration increases because of the release of bound TNS molecules from the serpin. Dissociation constant $K_{\mathrm{D}}$ for the antithrombin-PAA complex was determined by nonlinear least-squares fittings of two or three averaged measurements of the decreasing fluorescence intensity to the quadratic binding eq $2,{ }^{17}$ in which $\Delta F_{\mathrm{MAX}} / F_{0}$ is a second fitted parameter. A single-site binding model was assumed for the antithrombin interacting with smaller PAAs, including PAA830 through PAA3450, whereas a 2:1 stoichiometry was used for PAA6200.

$$
\begin{aligned}
& \frac{\Delta F}{F_{0}}=\left(\frac{\Delta F_{\mathrm{MAX}}}{F_{0}}\right)\left\{\left([\mathrm{AT}]_{0}+\left[\mathrm{PAA}_{0}+K_{\mathrm{D}}\right)-\right.\right. \\
& \left.\quad\left[\left([\mathrm{AT}]_{0}+[\mathrm{PAA}]_{0}+K_{\mathrm{D}}\right)^{2}-4[\mathrm{AT}]_{0}[\mathrm{PAA}]_{0}\right\}^{1 / 2} / 2[\mathrm{AT}]_{0}\right]
\end{aligned}
$$


Salt-Dependence of Equilibrium Dissociation Constants. The number of charge-charge interactions between antithrombin and PAA were determined by measurements of the equilibrium dissociation constants $K_{\mathrm{D}}$ at varying sodium concentrations. Fluorescence titrations of PAA binding to the antithrombin-TNS complex were conducted in $20 \mathrm{mM}$ sodium phosphate buffer, $\mathrm{pH}$ 6.0, containing $0.1 \mathrm{mM}$ EDTA and $0.1 \%$ PEG8000, at $25^{\circ} \mathrm{C}$ and in the presence of either 20, 35, 50, or $75 \mathrm{mM} \mathrm{NaCl}$. The ionic strength of the buffer increases from 0.025 to 0.1 as the salt concentration reaches $75 \mathrm{mM}$. The number of charge-charge interactions between PAA and antithrombin $(Z)$ was calculated from the slope of the linear plot of $\log K_{\mathrm{D} \text {,OBS }}$ vs $\log \left[\mathrm{Na}^{+}\right]$, according to eq 3 , whereas the nonionic contribution to the interaction was obtained from the intercept on the $y$-axis. ${ }^{6,8,18}$

$$
\log K_{\mathrm{D}}=\log K_{\mathrm{D}, \mathrm{NON}-\mathrm{IONIC}}+Z \psi \log \left[\mathrm{Na}^{+}\right]
$$

in which $K_{\mathrm{D}, \mathrm{NON}-\mathrm{IONIC}}$ is the dissociation constant at $\left[\mathrm{Na}^{+}\right]=1$ $\mathrm{M}, Z$ is the number of charge-charge interactions, and $\Psi$ is the fraction of monovalent counterions released per negative charge following PAA binding to antithrombin and is estimated to be 0.82 .

Competitive Binding of LAH to Antithrombin in the Presence of PAA3450. The equilibrium dissociation constant $K_{\mathrm{D}}$ of the antithrombin-LAH complex was measured at fixed concentrations of PAA3450 by monitoring the decrease in TNS fluorescence as a function of increasing LAH concentration at $25^{\circ} \mathrm{C}$. Titrations were performed in $20 \mathrm{mM}$ sodium phosphate buffer, pH 6.0, containing $20 \mathrm{mM} \mathrm{NaCl}, 0.1 \mathrm{mM}$ EDTA, and $0.1 \%$ PEG8000. The concentration of the competitor PAA3450 ranged from 4 to $12 \mu \mathrm{M}$, corresponding to a saturation of antithrombin ranging from 72 to $90 \%$. The decrease in fluorescence was analyzed in the same manner as above, using eq 2 to obtain the apparent equilibrium dissociation constant $K_{\mathrm{LAH}, \mathrm{app}}$.

\section{Competitive Binding of PAA3450 to Antithrombin in} the Presence of EFGH". Competitive binding of PAA3450 to antithrombin was studied in the presence of the tetrasaccharide EFGH" by measuring the residual thrombin activity. The protease activity was measured under pseudo-first-order conditions with $300 \mathrm{nM}$ antithrombin, $30 \mathrm{nM}$ thrombin, 100 nM PAA3450, and seven different concentrations of EFGH", ranging from 1 to $20 \mu \mathrm{M}$ in $20 \mathrm{mM}$ sodium phosphate buffer, $\mathrm{pH} 6.0$, containing $20 \mathrm{mM} \mathrm{NaCl}, 0.1 \mathrm{mM}$ EDTA, and $0.1 \%$ PEG8000. Concentrations of EFGH" ranged from 0.2 to 4.3 times that of the $K_{\mathrm{D}}$ of the competitor-antithrombin interaction. Fitting of the exponential decay of residual thrombin activity, determined after various times, yielded the pseudofirst-order rate constant $k_{\mathrm{OBS}}$ of protease inhibition. For the assumed case of competitive binding of EFGH" and PAA3450 at the pentasaccharide-binding site (PBS) of antithrombin, the Dixon-Webb relationship 4 was used to calculate the apparent dissociation constant $\left(K_{\mathrm{PAA}, \text { calc }}\right)$ of the antithrombin-PAA3450 complex.

$$
K_{\mathrm{PAA}, \text { calc }}=K_{\mathrm{PAA}}\left(1+\frac{\left[\mathrm{EFGH}^{\prime \prime}\right]_{0}}{K_{\mathrm{EFGH}^{\prime \prime}}}\right)
$$

in which $K_{\mathrm{PAA}}=1.3 \mu \mathrm{M}$, and $K_{\mathrm{EFGH}}=4.5 \mu \mathrm{M}$. Thus, the pseudo-first-order rate constant was calculated using $k_{\mathrm{OBS} \text {,calc }}$ $=k_{\mathrm{UNCAT}}[\mathrm{AT}]+k_{\mathrm{PAA}}[\mathrm{AT} / \mathrm{PAA}]$. In this equation, independently measured values of the uncatalyzed $\left(k_{\mathrm{UNCAT}}=570 \mathrm{M}^{-1} \mathrm{~s}^{-1}\right)$ and catalyzed $\left(k_{\mathrm{PAA}}=196000 \mathrm{M}^{-1} \mathrm{~s}^{-1}\right)$ rate constants were used, ${ }^{13}$ whereas [AT/PAA] was determined using the quadratic binding equation with the calculated dissociation constant $K_{\mathrm{PAA} \text {,calc. }}$.

Modeling of Antithrombin and PAA. Molecular modeling studies were performed using Sybyl 6.9.2 (Tripos Associates, St. Louis, MO) operating on a Tezro workstation (Silicon Graphics) in an Irix 6.5 operating system. The X-ray crystal structure of antithrombin $(2 a n t)^{19}$ was acquired from Research Collaboratory for Structural Bioinformatics (RCSB) (http:// www.rcsb.org/pdb/). The inhibitory monomer chain I was extracted from the dimeric structure and used as a model for the "native" conformation. Residues $34-42$, absent in the monomer, were not reengineered into the polypeptide chains because previous biochemical studies do not implicate these amino acids as important for heparin binding. Individual atoms were assigned Gasteiger-Hückel charges, and the polypeptide chain was minimized using a Tripos force field until a terminating gradient of $0.5 \mathrm{kcal} / \mathrm{mol} / \AA$ was reached.

The molecular structure of PAA with 33 carboxylic acid groups, representing PAA2280, was assembled in the small molecule builder module of Sybyl 6.9.2. Calculations were performed with a Tripos force field using Gasteiger-Hückel charges in an aqueous environment simulated with a dielectric constant of 80 . The initial torsional relaxation of the built structure was performed with 100 iterations of a steepestdecent minimization. The overall energy was reduced with a terminating gradient of $0.05 \mathrm{kcal} / \mathrm{mol} / \mathrm{A}$. The simulated annealing module implemented in Sybyl 6.9.2 was used to generate 10 different conformations of the polymer to simulate its structural diversity. During the simulated annealing, the polymer was heated to $700 \mathrm{~K}$ for $1000 \mathrm{fs}$, then cooled to $200 \mathrm{~K}$ within $1000 \mathrm{fs}$. These cycles were repeated 5 to 50 times in steps of 5 to generate 10 different conformations of PAA, which were used in docking studies.

Docking. The genetic algorithm of the FlexiDock module in Sybyl 6.9.2 was employed to search the plausible binding structures of PAA conformations in the heparin-binding site (HBS) of native antithrombin. Docking was carried out starting from an arbitrarily chosen position of the ligand in the HBS. The binding site was defined as residues within a distance of $4 \AA$ Arom Lys11, Arg13, Arg46, Arg47, Lys114, Lys125, Arg129, Arg132, Lys133, and Lys136, residues known to play an important role in heparin binding. The side chains of these amino acids and the PAA polymer were allowed torsional flexibility in the docking process, whereas the protein backbone and remaining bonds were held rigid. Atomic charges were calculated using the Gasteiger-Hückel protocol. No additional constraints, such as the explicit formation of a hydrogen bond between selected donor-acceptor sites, were introduced.

The initial parameters of the genetic algorithm were set to work on 10 islands with a population of 100 chromosomes each. Compared to 10 separate docking calculations, this approach allows for the interchange of high-fitness chromosomes between the islands, which decreases the likelihood for one island population to converge to a local minimum. To access a broad range of possible solutions, initial populations were generated within $6 \AA$ of the starting PAA coordinates. After 100000 generations, Flexidock provided between 20 and 200 solutions (1-20 for each island). For final consideration, 10 plausible solutions for each of the starting structures were selected and minimized to eliminate bad electronic and/or steric contacts.

Scoring. Hydropathic interaction (HINT, EduSoft LC, Ashland, VA) analysis is a computerized technique that quantitates nonpolar-nonpolar, polar-polar, and nonpolarpolar interactions between a ligand and its receptor. ${ }^{20,21}$ Briefly, HINT is not a statistical method nor is it a force field, but it employs parameters determined from solvent transfer experiments and thus is expected to better predict interactions at a molecular level. HINT calculates an interaction score between every intermolecular pair of atoms, and each such interaction score is added up to give the total intermolecular interaction score. HINT also searches for an interaction descriptor that qualifies each score as arising from a hydrogenbond, a van der Waals, or a Coulomb-type interaction.

HINT analysis was used to deduce antithrombin-PAA interactions simulated by modeling and docking experiments. Antithrombin was assigned HINT parameters from a generic dictionary obtained from a study of proteins. For antithrombin and PAA2280, only polar hydrogens were explicitly used in partitioning. Following calculations, HINT provides atomiclevel interaction scores for the docked complex. The atomiclevel HINT scores obtained were summed to derive scores at the amino acid level. 


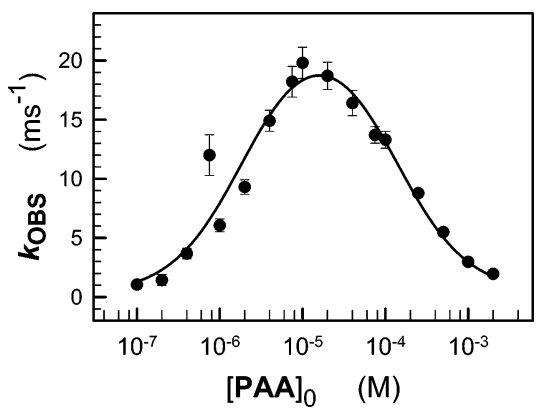

Figure 2. Dependence of antithrombin inhibition of thrombin on the concentration of PAA3450. The pseudo-first-order rate constant $\left(k_{\mathrm{OBS}}\right)$ of the thrombin inhibition was measured over a wide range of concentrations, from $100 \mathrm{nM}$ to $2 \mathrm{mM}$ PAA3450, at $\mathrm{pH} 6.0, I$ 0.025, and $25{ }^{\circ} \mathrm{C}$. The solid line represents the nonlinear regression fit of the data to eq 1 , which describes proteinase inhibition by antithrombin through the formation of a ternary complex. ${ }^{22}$

\section{Results}

Acceleration of Thrombin Inhibition by PAA2280. To test whether the PAA-promoted acceleration of antithrombin inhibition of thrombin originates from the bridging mechanism, the pseudo-first-order rate constant of thrombin inhibition $\left(k_{\mathrm{OBS}}\right)$ was measured over a wide range of PAA2280 concentrations at $\mathrm{pH} 6.0, I$ 0.025 , and $25{ }^{\circ} \mathrm{C}$. The $k_{\mathrm{OBS}}$ value increases gradually as the concentration of PAA2280 increases from 0.1 to $\sim 10 \mu \mathrm{M}$, as expected, indicating saturation of the inhibitor binding sites (Figure 2). At higher concentrations, $k_{\text {OBS }}$ plateaus and then decreases in a manner mirroring the ascending profile. This decrease in the rate of inhibition occurs because at high enough PAA concentrations, the binding of PAA to thrombin (T) starts to compete with its binding to antithrombin, eventually resulting in the preponderance of nonproductive binary complexes (AT-PAA + T-PAA) over the productive ternary complex $(\mathrm{AT}-\mathrm{PAA}-\mathrm{T}){ }^{22}$

The bell-shaped curve in Figure 2 is characteristic for the formation of a ternary complex. The data can be fitted to a model in which both AT-PAA and T-PAA complexes play a role in the overall rate of reaction (eq 1). Nonlinear regression analysis resulted in a secondorder rate constant of $83300 \pm 4600 \mathrm{M}^{-1} \mathrm{~s}^{-1}$ for the inactivation of thrombin by the antithrombin-PAA complex $\left(k_{\mathrm{ACT}}\right)$ and a dissociation constant of $109 \pm 23$ $\mu \mathrm{M}$ for the thrombin-PAA interaction $\left(K_{\mathrm{T}, \mathrm{PAA}}\right)$. The $k_{\mathrm{ACT}}$ value determined in this indirect method compares favorably with the value of $69000 \pm 3000 \mathrm{M}^{-1} \mathrm{~s}^{-1}$ determined in our previous direct measurements. ${ }^{13}$

Equilibrium Binding of LAH to Antithrombin in the Presence of PAA3450. To determine whether PAA molecules bind to antithrombin at the same site as does heparin, we performed competitive binding of LAH to antithrombin in the presence of PAA3450. We chose LAH instead of high-affinity heparin because the affinities of LAH and PAA are comparable, and thus, ensure the reliability of the competitive binding experiment.

If PAA interacts with the HBS, it is expected to compete with LAH in a predictable competitive manner described by the Dixon-Webb relationship. The apparent dissociation constant of the LAH-antithrombin interaction $\left(K_{\mathrm{LAH}, \mathrm{app}}\right)$ in the presence of several concen-

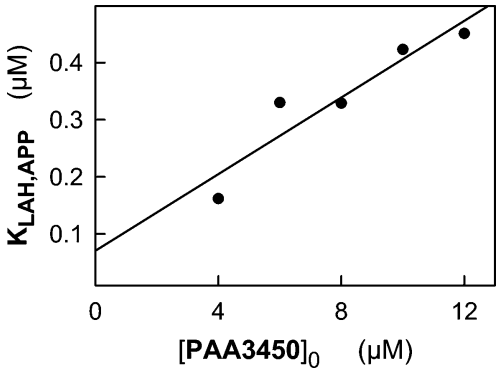

Figure 3. Dependence of the apparent equilibrium dissociation constant of the LAH-antithrombin interaction $\left(K_{\mathrm{LAH}, \mathrm{app}}\right)$ on the concentration of added PAA3450 at $\mathrm{pH} 6.0, I 0.025$, and $25{ }^{\circ} \mathrm{C}$. The solid line represents the linear regression fit of the data. Linear dependence in a predictable manner suggests competitive binding (see Methods and Results for further details).

trations of PAA3450 was measured by fluorescence titrations using TNS as the probe of binding, as described earlier (Figure 3). The initial fluorescence intensity decrease at each concentration PAA3450 tested was consistent with the $K_{\mathrm{PAA}}$ of the interaction (not shown). At the highest concentration of PAA3450 $(12 \mu \mathrm{M})$, the initial decrease in fluorescence was $-35 \%$, thus leaving a signal of $65 \%$ for the titration of LAH.

Figure 3 shows the change in $K_{\mathrm{LAH}, \text { app }}$ as a function of PAA3450 concentration. The linear dependence of $K_{\mathrm{LAH} \text {,app }}$ on the concentration of the activator suggests the competitive binding of LAH and PAA3450 to the HBS. Furthermore, the $y$-intercept of the linear regression analysis, corresponding to the equilibrium dissociation constant $\left(K_{\mathrm{LAH}}\right)$ of the LAH-antithrombin interaction, gives a value of $71 \mathrm{nM}$. This value compares favorably with the $K_{\mathrm{LAH}}$ determined independently under these conditions $(41 \pm 22 \mathrm{nM}){ }^{22}$ The slope of the regression line, which corresponds to the ratio of the dissociation constants of the competitors according to the Dixon-Webb equation $\left(K_{\mathrm{LAH}} / K_{\mathrm{PAA} 3450}\right)$, was found to be $0.034 \pm 0.007$. Using the $K_{\mathrm{LAH}}$ value determined independently, the equilibrium dissociation constant of the PAA3450-antithrombin interaction $\left(K_{\text {PAA3450 }}\right)$ was calculated to be $1.2 \mu \mathrm{M}$, which compares favorably with the value determined in direct titration $(1.3 \mu \mathrm{M}){ }^{13}$

PAA-Accelerated Inhibition of Thrombin in the Presence of EFGH". To test whether PAA interacts with PBS, we determined the influence of the tetrasaccharide EFGH" on the PAA-promoted antithrombin inhibition of thrombin. Previously, saccharide activator $\mathrm{EFGH}^{\prime \prime}$ has been shown to interact with the PBS with an affinity of $4.5 \pm 0.2 \mu \mathrm{M}$ at $\mathrm{pH} 6.0, I 0.025$, and 25 ${ }^{\circ} \mathrm{C} .{ }^{23}$ If PAA3450 interacts with the PBS of antithrombin, the presence of tetrasaccharide EFGH" is expected to decrease the PAA-promoted inhibition of thrombin in a manner predicted by the Dixon-Webb relationship. It is important to note that the tetrasaccharide minimally affects the rate of antithrombin inhibition of thrombin because polysaccharides greater than 16-18 residues are necessary for the formation of ternary complex with antithrombin and thrombin. ${ }^{6,7}$

The pseudo-first-order rate constant of thrombin inhibition $\left(k_{\mathrm{OBS}}\right)$ with $100 \mathrm{nM}$ PAA3450 decreased continuously from $k_{\mathrm{OBS}}=3.93 \mathrm{~ms}^{-1}$ in the presence of $1 \mu \mathrm{M}$ EFGH" to $0.7 \mathrm{~ms}^{-1}$ in the presence of $20 \mu \mathrm{M}$ $\mathrm{EFGH}^{\prime \prime}$ (Figure 4). This decrease in $k_{\mathrm{OBS}}$ with increasing $\mathrm{EFGH}^{\prime \prime}$ indicates competitive binding of the ligands in 


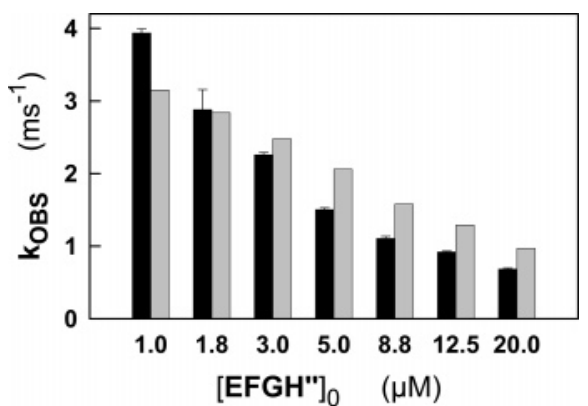

Figure 4. Dependence of the observed pseudo-first-order rate constant $\left(k_{\mathrm{OBS}}\right)$ of PAA3450-promoted antithrombin inhibition of thrombin on the concentration of added heparin tetrasaccharide $\mathrm{EFGH}^{\prime \prime}$ at $\mathrm{pH}$ 6.0, I 0.025 , and $25{ }^{\circ} \mathrm{C}$. A fixed concentration of $100 \mathrm{mM}$ PAA3450 was used to detect competition by EFGH". Tetrasaccharide EFGH" is a four-residue sequence derived from pentasaccharide DEFGH with small modifications in residue $\mathrm{H}$ (hence it is labeled as $\mathrm{H}^{\prime \prime}$ ) which do not affect the mechanism of binding. The first-order rate constants $\left(k_{\mathrm{OBS}}\right)$ were determined from the exponential decrease of residual thrombin activity (shown as black bars) as described in Materials and Methods. The gray bars represent calculated values of $k_{\mathrm{OBS}}$ using the affinities of antithrombin with PAA3450 and $\mathrm{EFGH}^{\prime \prime}\left(K_{\mathrm{PAA}}\right.$ and $\left.K_{\mathrm{EFGH}}\right)$, the PAApromoted second-order thrombin inhibition rate constant $k_{\text {PAA, }}$, and the Dixon-Webb relationship for competitive inhibition. The comparable decrease of both the observed and calculated rate constant values with increasing EFGH" concentration indicates the competitive binding of the tetrasaccharide and PAA. Error shown on the observed rate constant bars are \pm 1 SE

the PBS. Thus, the apparent dissociation constants $K_{\text {PAA,app }}$ of antithrombin-PAA complexes can be calculated on the basis of the Dixon-Webb relationship, which allows for the prediction of the apparent pseudofirst-order rate constant at each $\left[\mathrm{EFGH}^{\prime \prime}\right]_{0}$ (see Materials and Methods). Figure 4 compares the calculated and observed pseudo-first-order rate constants at various concentrations of EFGH". Within the limits of experimental error, the close correspondence indicates competitive binding of PAA and EFGH" in the PBS of antithrombin.

Ionic and Non-Ionic Interactions between PAA and Antithrombin. According to the counterion condensation theory, the binding of a polyelectrolyte (PE), such as PAA, to antithrombinfollows an ion-exchange process, which proceeds favorably because of the release of $\mathrm{M}^{+}$cations..$^{18,24}$

$$
\mathrm{AT}^{Z+}+\mathrm{PE} \stackrel{K_{\mathrm{D}}}{\longleftrightarrow} \mathrm{AT} / \mathrm{PE}+Z \Psi \mathrm{M}^{+}
$$

In this process, the formation of $Z$ electrostatic interactions between $\mathrm{AT}$ and $\mathrm{PE}$ is accompanied by the displacement of $\Psi Z$ condensed counterions, in which $\Psi$ is the fraction of cations released to the bulk solution per negative charge. Thus, the ion-exchange process is highly susceptible to the screening effects of salt, which can be monitored in a predictable manner (eq 3). We determined the salt-dependence of the binding constants for three representative PAA polymers, PAA1500, PAA2280, and PAA6200, to understand the nature of their interactions with antithrombin. A consistent decrease in binding affinity with increasing salt concentration was noted for all three PAAs (Figure 5). Linear regression of the data using eq 3 produced slopes of 3.9 (PAA1500), 4.1 (PAA2280), and 4.0 (PAA6200) (Table

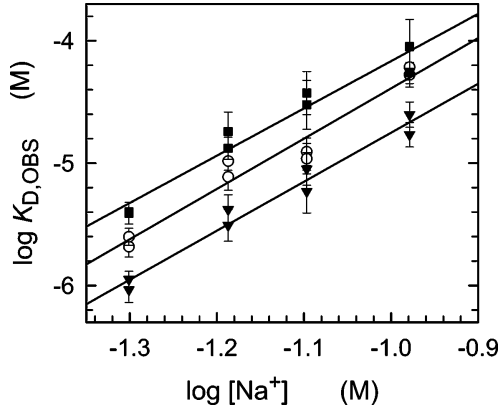

Figure 5. Dependence of the equilibrium dissociation constants $\left(K_{\mathrm{D}, \mathrm{OBS}}\right)$ of antithrombin-PAA interaction on the sodium concentration $\left[\mathrm{Na}^{+}\right]$for PAA1500 (四, PAA2280 (O), and PAA6200 ( $\boldsymbol{\nabla})$. Solid lines show the linear least-squares fits of the data by eq 3 . The dissociation constants were determined by the titration of $0.95 \mu \mathrm{M}$ antithrombin in $20 \mathrm{mM}$ sodium phosphate, 0.1 mM EDTA, 0.1\% PEG8000, and 5.5 mM TNS with $0.2-2 \mathrm{mM}$ solutions of PAA (see Materials and Methods for details).

Table 1. Thermodynamic Parameters for Antithrombin-PAA Interaction as Determined from the Salt-Dependence of the Equilibrium Dissociation Constant at $\mathrm{pH}$ 6.0, I 0.025, and 25 ${ }^{\circ} \mathrm{C}^{a}$

\begin{tabular}{lcccccc}
\hline & slope & $\mathrm{Z}$ & intercept & $\begin{array}{c}K_{\mathrm{D}, \text { nonionic }} \\
(\mathrm{mM})\end{array}$ & $\begin{array}{c}\Delta G_{\text {ionic }}^{0} \\
(\mathrm{~kJ} / \mathrm{mol})\end{array}$ & $\begin{array}{c}\Delta G_{\text {nonionic }}^{0} \\
(\mathrm{~kJ} / \mathrm{mol})\end{array}$ \\
\hline PAA1500 & 3.9 & 4.7 & -0.31 & 495 & 30.5 & 1.7 \\
PAA2280 & 4.1 & 5.0 & -0.28 & 523 & 30.9 & 1.3 \\
PAA6200 & 4.0 & 4.9 & -0.75 & 179 & 30.0 & 4.3 \\
DEFGH $^{b}$ & 4.2 & 5.2 & -4.92 & 0.012 & 38.9 & 28.0 \\
\hline
\end{tabular}

${ }^{a}$ The number of salt bridges $(Z)$ and the nonionic contribution to $K_{\mathrm{D} \text {,OBS }}\left(K_{\mathrm{D} \text {,nonionic }}\right)$ were determined by the linear regression of the dependence $\log K_{\mathrm{D} \text {,oBs }}$ on $\log \left[\mathrm{Na}^{+}\right]$, measured at $\mathrm{pH} 6.0, I$ 0.025 , and $25{ }^{\circ} \mathrm{C} .{ }^{b}$ Data taken from ref 8 .

1). These slopes correspond to $\sim 4.7,5.0$, and 4.9 chargecharge interactions, respectively, between antithrombin and PAA. Thus, the number of ionic interactions formed is nearly independent of the length of polymer.

The intercepts of the double logarithmic plots were found to be $-0.31,-0.28$, and -0.75 for PAA1500, PAA2280, and PAA6200, respectively (Table 1). The intercepts correspond to the equilibrium dissociation constants at $\left[\mathrm{Na}^{+}\right]=1 \mathrm{M}$, thus yielding the contribution of nonionic forces to the binding affinity $K_{\mathrm{D} \text {,nonionic. }}$ The nonionic dissociation constants measured for the antithrombin-PAA complexes were found to be 495,523 , and $179 \mathrm{mM}$ for PAA1500, PAA2280, and PAA6200, respectively.

Modeling of Antithrombin-PAA Interactions. To identify the antithrombin residues most likely involved in PAA binding, we employed computerized molecular modeling using Flexidock, a genetic algorithm-based docking protocol that allows nearly full flexibility of rotatable bonds in both the ligand and the protein. In an ideal scenario, docking into a known protein-binding pocket is expected to give one optimal solution. However, PAA is a rather flexible molecule that can adopt a number of different conformations in aqueous solution. Thus, chemical intuition suggests several binding modes, which may not differ significantly in terms of energy. Therefore, a statistical approach was chosen, which involved 10000 PAA structures docking into the native (chain I of RCSB file 2ant) form of antithrombin, each being allowed to evolve over 100000 iterations. Following the docking, 10 best Flexidock solutions were 


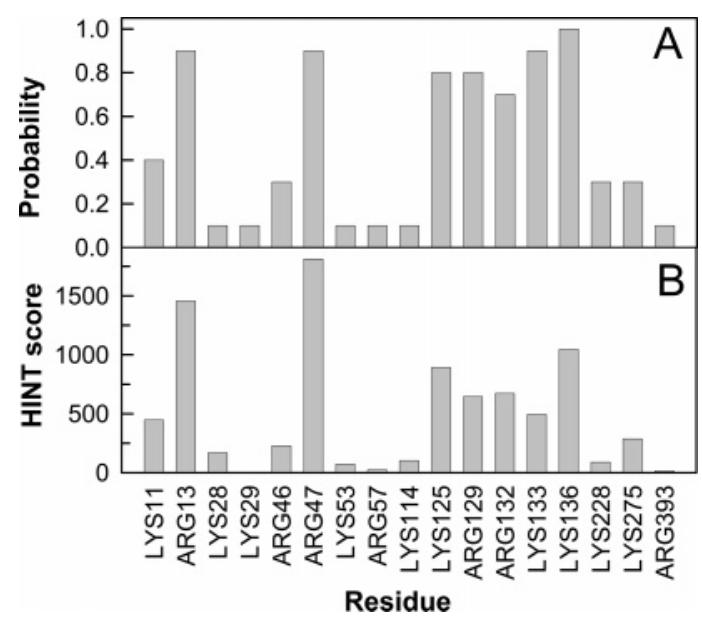

Figure 6. HINT analysis of antithrombin-PAA interaction. (A) Average probability of charged amino acid residues of antithrombin to occur in PAA interaction (calculated from final 10 optimal docking solutions). (B) Average residue level HINT score for interaction with PAA2280. Nearly all positively charged residues of the HBS contribute to the interaction with PAA, yet predominant contributions arise from residues $\mathrm{R} 13$ and R47, followed by the residues Lys125, Arg129, Arg132, Lys133, and Lys136 (see text for details).

selected, using energetic considerations, as representing the most probable PAA-antithrombin structures and scored using HINT.

Although HINT is known to identify and quantify intermolecular nonpolar interactions, for the 10 representative PAA-native AT complexes, the nonpolar HINT scores fluctuated around zero (not shown). This suggests that the molecular interaction between PAA and native AT minimally involves hydrogen bonding or van der Waals-type forces. In contrast, electropositive residues of the HBS show HINT scores in the range of 200-2000. Atomic-level analysis of the HINT interaction profile (not shown) suggests that these scores arise from the favorable interaction of positively charged side chains with the negatively charged carboxylate groups of PAA.

HINT implicates several electropositive residues of native antithrombin in binding to PAA, including Lys11, Arg13, Arg46, Arg47, Lys53, Arg57, Lys114, Lys125, Arg129, Arg132, Lys133, and Lys136 (Figure 6A). These residues belong to both the $\mathrm{PBS}$ and the extended heparin-binding site (EHBS). Yet, not all are equally important. Calculation of the relative probability of occurrence of individual residues (Figure 6A) shows that seven residues, Arg13, Arg47, Lys125, Arg129, Arg132, Lys133, and Lys136, are likely to be most important. These residues are expected to contribute most of the binding energy involved in the interaction, with their relative order of importance decreasing in the following order: Arg47, Arg13, Lys136, Lys125, Arg132, Arg129, and Lys133 (Figure 6B). The overall HINT scores for the final 10 solutions ranged from 5389 to 9134 with an average of $7480 \pm 1260$.

\section{Discussion}

The fact that accelerated thrombin inhibition is brought about primarily through the bridging mechanism ${ }^{6,7}$ and minimally through conformational change in antithrombin has one major implication. It suggests that molecules capable of simultaneously binding throm- bin and antithrombin will enhance the inhibition rate, 519 even if the serpin exists in its native, "unactivated" 520 state. Our previous discovery that PAAs, molecules 521 containing only carboxylic acid groups, can possibly 522 serve as such "bridges" led us to speculate that it should 523 be feasible to design orally available heparins using a 524 prodrug approach. ${ }^{13}$ However, a problem that was 525 apparent in the initial studies was the poor affinity of 526 PAA for antithrombin. Several explanations are possible 527 for the lack of tight binding, including (i) the highly 528 flexible nature of PAA resulting in high entropic cost of 529 binding, (ii) the possibility that PAA recognizes a site 530 in antithrombin different from the HBS, and (iii) the 531 possibility that carboxylate groups of PAA do not 532 effectively mimic the sulfate groups of sulfated polysac- 533 charides. In addition, although PAA promoted anti- 534 thrombin inhibition of thrombin $\left(k_{\mathrm{CAT}} / k_{\mathrm{UNCAT}}\right)$ in a chain 535 length-dependent manner (see Figure 1), ${ }^{13}$ it was not 536 clear whether these molecules provided a template for 537 the formation of a T-PAA-AT ternary complex, espe- 538 cially because even PAA830, with a length of only $29 \AA 589$ in its most extended form, which is far below the optimal 540 length of $\sim 100 \AA$ necessary for bridging, displayed some 541 accelerating abilities. To answer these questions and to 542 advance the design of PAA-based heparin mimics, we 543 studied the molecular interaction of PAA with anti- 544 thrombin and thrombin in detail. Most studies were 545 performed at $\mathrm{pH} 6.0$, rather than at the more physi- 546 ologically relevant $\mathrm{pH} 7.4$, because the higher affinity 547 observed at the lower $\mathrm{pH}$ introduces greater reliability. 548 More importantly, we have shown earlier that results 549 under the two conditions parallel each other, thus 550 allowing ready extrapolation. ${ }^{8,25}$

To test whether PAAs provide a template for the serpin and the proteinase to interact, we determined the dependence of the pseudo-first-order rate constant $k_{\text {OBS }}$ of thrombin inhibition as a function of the concentration of PAA2280 over nearly 3 log units. A bellshaped profile, similar to that observed for full-length heparin, ${ }^{22}$ was observed, which is characteristic of inhibition through the bridging mechanism (Figure 2). A major difference between the bell-shaped profiles for heparin and PAA was the concentration at which the phenomenon could be observed in each case, with the PAA profile shifted to the higher concentration side by $\sim 2-3 \log$ units. Yet, the bell-shaped profile rigorously shows that PAA2280 simultaneously interacts with thrombin and antithrombin. The observation that thrombin can bind a polycarboxylate ligand is interesting but not unexpected, especially because thrombin has been known to bind many different sulfated structures and is also known to interact well with ligands containing phosphate groups. ${ }^{26}$ The interaction of thrombin with these polyanions is thought to be primarily electrostatic and nonspecific, ${ }^{27}$ thus explaining its ability to bind a purely carboxylic acid domain. Regressional analysis of the bell-shaped profile yields an equilibrium dissociation constant of the thrombin-PAA interaction $\left(K_{\mathrm{T}, \mathrm{PAA}}\right)$ of $109 \pm 23 \mu \mathrm{M}$ at $\mathrm{pH}$ 6.0. In comparison, thrombinheparin interaction has an affinity of $4-10 \mu \mathrm{M}$ at $\mathrm{pH}$ 7.4. ${ }^{27,28}$ Sulfated molecules bind thrombin in a domain called exosite II, which is composed of positively charged residues Arg93, Arg101, Arg165, Arg233, Lys235, Lys236, and Lys240 spread over several helices and turns. ${ }^{28}$ 
Thus, the weaker affinity of the PAA-thrombin interaction likely originates from fewer ion-pairs interactions being formed during thrombin complexation with PAA as compared to the $\sim 5$ ion-pairs formed with heparin. ${ }^{27}$

To determine whether PAA binds antithrombin in the HBS, competitive binding studies were performed with LAH and tetrasaccharide EFGH". The HBS in antithrombin consists of two domains: ${ }^{11}$ (1) the PBS formed by residues Lys11 and Arg13 of the N-terminus, Arg46, Arg47, and Trp49 of helix A, and Lys114, Phe121, Lys125, and Arg129 of helices P and D and (2) the EHBS formed by residues Arg132, Lys133, and Lys136. Whereas EFGH" interacts with the PBS alone, ${ }^{8,23} \mathrm{LAH}$ interacts with both PBS and EHBS. ${ }^{22}$ Fluorescence titrations of the antithrombin-TNS complex with LAH in the presence of increasing concentrations of PAA3450 show a linear decrease in LAH binding affinity in accordance with the Dixon-Webb relationship (Figure 3 ), thus suggesting that both LAH and PAA engage identical binding sites. Likewise, EFGH"'-induced (Figure 4) deceleration in the pseudo-first-order rate of PAApromoted antithrombin inhibition of thrombin suggests that the tetrasaccharide interferes with antithrombinPAA interaction. Thus, PAA interacts with both the PBS and the EHBS in antithrombin.

To understand whether carboxylic acid groups can effectively mimic sulfate groups, the thermodynamic characteristics of antithrombin-PAA interaction were studied by measurements of the dissociation constant $\left(K_{\mathrm{D} \text {,app }}\right)$ as a function of salt concentration. Analysis of the results, according to the counterion condensation theory, yields the number of salt bridges $(Z)$ involved in PAA binding as well as the ionic and nonionic contributions $\left(\Delta G_{\text {ionic }}^{0}\right.$ and $\Delta G_{\text {nonionic }}^{0}$ ) to the standard free energy of binding $\left(\Delta G_{\mathrm{OBS}}^{0}\right) .^{6,18,24}$ The results for three PAAs are consistent and identical, indicating a chain-length-independent interaction profile with antithrombin. Nearly four $\mathrm{Na}^{+}$ions are released in the binding of PAA with antithrombin. In other words, five salt bridges are formed in the process, resulting in an electrostatic standard free energy of binding $\left(\Delta G_{\text {ionic }}^{0}\right)$ of $30.0 \mathrm{~kJ} / \mathrm{mol}$. In contrast, the pentasaccharide DEFGHantithrombin interaction involves $\sim 6.5$ salt bridges, corresponding to a $\Delta G_{\text {ionic }}^{0}$ of $38.9 \mathrm{~kJ} / \mathrm{mol}$ under nearly identical conditions. ${ }^{8,25}$ This suggests an identical 6.0$\mathrm{kJ} / \mathrm{mol}$ contribution per salt bridge formed, irrespective of whether it is PAA or heparin pentasaccharide. Thus, the ionic free energy of binding involved in polycarboxylate-antithrombin interaction is comparable to that of polysulfate-antithrombin interaction.

However, the equivalent number of salt bridges formed in the two interactions is more likely to be fortuitous and does not suggest the formation of identical salt bridges. One, pentasaccharide DEFGH releases all five $\mathrm{Na}^{+}$ions from the $\mathrm{PBS},{ }^{8}$ whereas our competitive binding studies suggest that PAA likely interacts with EHBS also. Two, pentasaccharide DEFGH induces a conformational change in the $\mathrm{HBS}, 6,8$ whereas our previous studies suggest that PAAs do not appear to induce conformational changes in the serpin. ${ }^{13}$ Thus, PAA is expected to interact with a set of positively charged amino acid residues in antithrombin that is different than that with which pentasaccharide DEFGH interacts. Previous work with full-length heparin dem- onstrates the formation of additional ionic interactions 647 with residues of the EHBS; ${ }^{29,30}$ thus one can expect that 648 PAA forms one or more salt bridges with this domain, 649 which is also supported by modeling studies (below). 650

A striking contrast between the interaction of car- 651 boxylates and sulfates is evident from the difference in 652 the contribution of nonionic forces to the binding energy. 653 Whereas the nonionic standard free energy of binding 654 $\left(\Delta G_{\text {nonionic }}^{0}\right)$ is $4.3 \mathrm{~kJ} / \mathrm{mol}$ for PAA6200-antithrombin 655 interaction, that for DEFGH and full-length heparin is 656 $28.0 \mathrm{~kJ} / \mathrm{mol} .^{6,8,30}$ The nonionic contribution measured in 657 the experimental setup represents all forces other than 658 ionic, which primarily include hydrogen-bonding and 659 van der Waals. Yet, we have previously shown, using 660 molecular modeling, that antithrombin interaction with 661 heparin-based activators minimally involves interaction 662 with the backbone atoms, ${ }^{31}$ an observation also sup- 663 ported by the X-ray crystal structure of the co-complex. ${ }^{32} 664$ This suggests that the groups that dominate the anti- 665 thrombin-heparin interaction are the anionic groups, 666 primarily sulfate, of the saccharide activator. Thus, the 667 difference in nonionic contribution between PAA and 668 sulfated activators originates from the difference in the 669 hydrogen-bonding capability of the lysines and arginines 670 of the HBS with sulfate and carboxylate groups. Our 671 work suggests that carboxylates are significantly weaker 672 than sulfates in their hydrogen-bonding capability. This 673 defect appears to be the single most factor in the weaker 674 affinity of PAAs and has major implications in the design of orally active activators.

To identify the positively charged residues of the HBS involved in PAA binding, the antithrombin-PAA interaction was modeled in silico. Because our previous work suggests that PAAs do not induce any major conformational changes in antithrombin, as judged by the absence of enhancement in intrinsic protein tryptophan fluorescence and by the marginal increase in the PAApromoted antithrombin inhibition of factor $\mathrm{Xa}$ at $\mathrm{pH}$ $7.4,{ }^{13}$ we performed docking and scoring studies with antithrombin in the native form (2ant). Statistical analysis suggests that seven residues are important for PAA binding. These include Arg13, Arg47, Lys125, Arg129, Arg132, Lys133, and Lys136. In contrast, Lys11, Arg46, and Lys114 do not appear to be important. It is interesting that the docking protocol implicates residues belonging to both the PBS and the EHBS, ${ }^{11}$ an observation that supports the competitive binding studies. In addition, modeling suggests that two of the three critical residues for the heparin binding of the PBS, Lys125 and Arg129, are likely to interact with PAA. These two residues, together with Lys114, form a crucial network that plays a dominant role in both heparin recognition and antithrombin conformational activation. ${ }^{33-36}$ This network functions cooperatively, and suboptimal interaction with one of the residues is likely to have profound implications on the binding of the other two. Furthermore, Lys114 interacts with the critical $3-\mathrm{OSO}_{3}{ }^{-}$group of residue $\mathrm{F}$ of the heparin pentasaccharide, contributing a massive $50 \%$ of the total $\Delta \mathrm{G}^{\circ}$. Thus, the absence of PAA interaction with Lys 114 is expected to be detrimental. In terms of the number of salt bridges, HINT analysis indicates strongly positive average scores with a high probability of occurrence for Arg47 and Arg13 and moderately positive average 
scores for Lys136, Lys125, Arg129, Arg132, and Lys133 (in that order). These seven residues contribute more than $95 \%$ of the total HINT score with an average probability of $86 \%$. Thus, the HINT data finds good support in the experimentally determined $Z$ value of the five salt bridges involved in the interaction.

The mechanistic study undertaken herein highlights important principles for the design of organic activators of antithrombin. Interestingly, this work with carboxylate-only molecules introduces more rules about strategies that will not succeed than rules about strategies that are likely to succeed. For example, despite the high activation achieved with carboxylate-only activators, this approach will not succeed because of a fundamentally impaired binding (absence of nonionic binding energy) of carboxylate groups. This conclusion rules out the possibility of an easy carboxylate-only-based prodrug approach. At the same time, the results highlight the need to retain critical sulfate groups in the antithrombin-binding domain, especially those interacting with Arg129, Lys125, and Lys114. The implementation of this design principle is feasible because of the successful simulation of the PAA-antithrombin complex coupled with HINT analysis. For example, selected carboxylate groups in the vicinity of Arg129, Lys125, and Lys114 can be replaced with sulfate groups, followed by the design of a backbone that is more rigid than that of PAA. This process, performed iteratively using HINT analysis, is expected to result in a new antithrombin activator that has sulfate groups interspersed with carboxylate groups. The limitation imposed by this alternating framework necessitates a completely different prodrug strategy for the introduction of oral activity. We speculate that a prodrug approach involving a dual protecting group, which simultaneously modifies appropriately positioned sulfate and carboxylate groups and hydrolyzes in vivo, ${ }^{37}$ will be necessary. These new principles of design for the antithrombin-binding domain may be utilized to similarly design a thrombin-binding domain. The results of this rational design strategy will be reported in the future.

Acknowledgment. We thank Prof. Steven T. Olson of University of Illinois - Chicago for his generous gift of purified low-affinity heparin and oligosaccharide EFGH". This work was supported by American Heart Association Mid-Atlantic affiliate (0256286U) and the National Heart, Lung and Blood Institute (RO1 HL069975).

\section{References}

(1) Björk, I.; Olson, S. T. Antithrombin: a bloody important serpin. In Chemistry and Biology of Serpins; Church, F. C., Cunningham, D. D., Ginsburg, D., Hoffman, M., Tollefsen, D. M., Stone, S. R., Eds.; Plenum Press: New York, 1997; pp 17-33.

(2) Gettins, P. G. W. Serpin structure, mechanism, and function. Chem. Rev. 2002, 102, 4751-4803.

(3) Olson, S. T.; Swanson, R.; Raub-Segall, E.; Bedsted, T.; Sadri, M.; Petitou, M.; Herault, J. P.; Herbert, J. M.; Björk, I. Accelerating ability of synthetic oligosaccharides on antithrombin inhibition of proteinases of the clotting and fibrinolytic systems. Comparison with heparin and low-molecular-weight heparins. Thromb. Haemostasis 2004, 92, 929-939.

(4) Bedsted, T.; Swanson, R.; Chuang, Y.-J.; Bock, P. E.; Bjork, I.; Olson, S. T. Heparin and calcium ions dramatically enhance antithrombin reactivity with factor IXa by generating new interaction exosites. Biochemistry 2003, 42, 8143-8152.

(5) Rezaie, A. R. Calcium enhances heparin catalysis of the antithrombin-factor Xa reaction by a template mechanism. J. Biol. Chem. 1998, 273, 16824-16827.
(6) Olson, S. T.; Björk, I.; Sheffer, R.; Craig, P. A.; Shore, J. D.; Choay, J. Role of the antithrombin-binding pentasaccharide in heparin acceleration of antithrombin-proteinase reactions. Resolution of the antithrombin conformational change contribution to heparin rate enhancement. J. Biol. Chem. 1992, 267, 12528-12538.

(7) Olson, S. T.; Björk, I. Predominant contribution of surface approximation to the mechanism of heparin acceleration of the antithrombin-thrombin reaction: Elucidation from salt concentration effects. J. Biol. Chem. 1991, 266, 6353-6364.

(8) Desai, U. R.; Petitou, M.; Björk, I.; Olson, S. T. Mechanism of heparin activation of antithrombin. Role of individual residues of the pentasaccharide activating sequence in the recognition of native and activated states of antithrombin. J. Biol. Chem. 1998, 273, 7478-7487.

(9) Hirsh, J.; Anand, S. S.; Halperin, J. L.; Fuster, V. Guide to anticoagulant therapy: heparin. Circulation 2001, 103, 29943018 .

(10) Fareed, J.; Hoppensteadt, D. A.; Bick, R. L. An update on heparins at the beginning of the new millennium. Semin. Thromb. Hemostasis 2000, 26, 5-21.

(11) Desai, U. R. New antithrombin-based anticoagulants. Med. Res. Rev. 2004, 24, 151-181.

(12) Petitou M.; van Boeckel, C. A. A. A synthetic antithrombin III binding pentasaccharide is now a drug! What comes next? Angew. Chem., Int. Ed. 2004, 43, 3118-3133.

(13) Monien, B. H.; Desai, U. R. Antithrombin activation by nonsulfated, nonpolysaccharide organic polymer J. Med. Chem. 2005, 48, 1269-1273.

(14) Church, F. C.; Treanor, R. E.; Sherrill, G. B., Whinna, H. Carboxylate polyanions accelerate inhibition of thrombin by heparin cofactor II. Biochem. Biophys. Res. Commun. 1987, 148, 363-368.

(15) Gunnarsson, G. T.; Desai, U. R. Designing small, nonsugar activators of antithrombin using hydropathic interaction analyses. J. Med. Chem. 2002, 45, 1233-1243.

(16) Meagher, J. L.; Olson, S. T.; Gettins, P. G. W. Critical role of the linker region between helix D and strand 2A in heparin activation of antithrombin. J. Biol. Chem. 2000, 275, 2698-2704.

(17) Olson, S. T.; Björk, I.; Shore, J. D. Kinetic characterization of heparin-catalyzed and uncatalyzed inhibition of blood coagulation proteinases by antithrombin. Methods Enzymol. 1993, 222, $525-560$.

(18) Manning, G. S. The molecular theory of polyelectrolyte solutions with applications to the electrostatic properties of polynucleotides. Q. Rev. Biophys. 1978, 11, 179-249.

(19) Skinner, R.; Abrahams, J.-P.; Whisstock, J. C.; Lesk, A. M.; Carrell, R. W.; Wardell, M. The 2.6 A structure of antithrombin indicates a conformational change at the heparin binding site. J. Mol. Biol. 1997, 266, 601-609.

(20) Cozzini, P.; Fornabaio, M.; Marabotti, A.; Abraham, D. J.; Kellogg, G. E.; Mozzarelli, A. Free energy of ligand binding to protein: evaluation of the contribution of water molecules by computational methods. Curr. Med. Chem. 2004, 11, 3093-3118.

(21) Meng, E. C.; Kuntz, I. D.; Abraham, D. J.; Kellogg, G. E. Evaluating docked complexes with the HINT exponential function and empirical atomic hydrophobicities. J. Comput.-Aided Mol. Des. 1994, 8, 299-306.

(22) Streusand, V. J.; Björk, I.; Gettins, P. G. W.; Petitou, M.; Olson, S. T. Mechanism of acceleration of antithrombin-proteinase reactions by low affinity heparin: Role of the antithrombin binding pentasaccharide in heparin rate enhancement. J. Biol. Chem. 1995, 270, 9043-9051.

(23) Gunnarsson, G. T.; Desai, U. R. Interaction of sulfated flavanoids with antithrombin: lessons on the design of organic activators. J. Med. Chem. 2002, 45, 4460-4470.

(24) Record, M. T., Jr.; Lohman, M. L.; De Haseth, P. Ion effects on ligand-nucleic acid interactions. J. Mol. Biol. 1976, 107, 145158.

(25) Desai, U. R.; Petitou, M.; Björk, I.; Olson, S. T. Mechanism of heparin activation of antithrombin: evidence for an induced-fit model of allosteric activation involving two interaction subsites. Biochemistry 1998, 37, 13033-13041.

(26) Buijsman, R. C.; Basten, J. E.; Dreef-Tromp, C. M.; van der Marel, G. A.; van Boeckel, C. A.; van Boom, J. H. Synthesis of heparin-like antithrombotics having perphosphorylated thrombin binding domains. Bioorg. Med. Chem. 1999, 7, 1881-1890.

(27) Olson, S. T.; Halvorson, H. R.; Björk, I. Quantitative characterization of the thrombin-heparin interaction. Discrimination between specific and nonspecific binding models. J. Biol. Chem. 1991, 266, 6342-6352.

(28) Carter, W. J.; Cama, E.; Huntington, J. A. Crystal structure of thrombin bound to heparin. J. Biol. Chem. 2005, 280, 27452749.

(29) Arocas, V.; Turk, B.; Bock, S. C.; Olson, S. T.; Björk, I. The region of antithrombin interacting with full-length heparin chains outside the high-affinity pentasaccharide sequence extends to Lys136 but not to Lys139. Biochemistry 2000, 39, 8512-8518. 
(30) Turk, B.; Brieditis, I.; Bock, S. C.; Olson, S. T.; Björk, I. The oligosaccharide side chain on Asn-135 of alpha-antithrombin, absent in beta-antithrombin, decreases the heparin affinity of the inhibitor by affecting the heparin-induced conformational change. Biochemistry 1997, 36, 6682-6691.

(31) Desai, UR.; Gunnarsson, G. Hydropathic interaction analysis of sequence-specific heparin pentasaccharide binding to antithrombin. Med. Chem. Res. 1999, 9, 643-655.

(32) Jin, L.; Abrahams, J.-P.; Skinner, R.; Petitou, M.; Pike, R. N.; Carrell, R. W. The anticoagulant activation of antithrombin by heparin. Proc. Natl. Acad. Sci. U.S.A. 1997, 94, 14683-14688.

(33) Schedin-Weiss, S.; Arocas, V.; Bock, S. C.; Olson, S. T.; Björk, I. Specificity of the basic side chains of Lys114, Lys125 and Arg129 of antithrombin in heparin binding. Biochemistry 2002, 41, 12369-12376.
(34) Schedin-Weiss, S.; Desai, U. R.; Bock, S. C.; Gettins, P. G. W.; 882 Olson, S. T.; Björk, I. The importance of lysine 125 for heparin 883 binding and activation of antithrombin. Biochemistry 2002, 41, 884 4779-4788.

(35) Desai, U. R.; Swanson, R. S.; Bock, S. C.; Björk, I.; Olson, S. T. 886 The role of arginine 129 in heparin binding and activation of 887 antithrombin. J. Biol. Chem. 2000, 275, 18976-18984. 888

(36) Arocas, V.; Bock, S. C.; Raja, S.; Olson, S. T.; Björk, I. Lysine 889 114 of antithrombin is of crucial importance for the affinity and 890 kinetics of heparin pentasaccharide binding. J. Biol. Chem. 2001, 891 276, 43809-43817.

(37) Roberts, J. C.; Gao, H.; Gopalsamy, A.; Kongsjahju, A.; Patch, 893 R. J. Neopentyl ester protecting groups for aryl sulfonic acids. 894 Tetrahedron Lett. 1997, 38, 355-358.

JM0503648 